June 2005

CERN-TH/2005-108

\title{
On the Tuning Condition of Split Supersymmetry
}

\author{
A. Delgado, G.F. Giudice \\ CERN, Theory Division, CH-1211 Geneva 23, Switzerland
}

\begin{abstract}
Split Supersymmetry does not attempt to solve the hierarchy problem, but it assumes a tuning condition for the electroweak scale. We clarify the meaning of this condition and show how it is related to the underlying parameters. Simple assumptions on the structure of the soft terms lead to predictions on $\tan \beta$ and on the physical Higgs mass.
\end{abstract}


Split Supersymmetry [1, 2, 3] does not attempt to give a natural explanation of the hierarchy problem but, as in the Standard Model, a parameter fine-tuning is assumed to obtain the correct breaking of electroweak symmetry. In this paper we want to show that, although the theory does not provide a dynamical explanation for the tuning, its existence leads to important information on the underlying parameters and on measurable physical quantities.

Let us start by considering the potential in Split Supersymmetry for the real and neutral Higgs field $h$, valid at energies lower than the squark and slepton mass scale $\tilde{m}$,

$$
V=\frac{m^{2}}{2} h^{2}+\frac{\lambda}{8} h^{4}
$$

The Higgs mass parameter $m^{2}$ satisfies to the RG equation

$$
\begin{aligned}
& 16 \pi^{2} \bar{\mu} \frac{d}{d \bar{\mu}} m^{2}=\left[6 \lambda-\frac{3}{2}\left(g^{\prime 2}+3 g^{2}\right)+3\left(\tilde{g}_{u}^{2}+\tilde{g}_{d}^{2}\right)+\tilde{g}_{u}^{\prime 2}+\tilde{g}_{d}^{\prime 2}+6 h_{t}^{2}\right] m^{2} \\
& -2\left[\left(\tilde{g}_{u}^{\prime 2}+\tilde{g}_{d}^{\prime 2}\right)\left(M_{1}^{2}+\mu^{2}\right)+3\left(\tilde{g}_{u}^{2}+\tilde{g}_{d}^{2}\right)\left(M_{2}^{2}+\mu^{2}\right)+2\left(\tilde{g}_{u}^{\prime} \tilde{g}_{d}^{\prime} M_{1}+3 \tilde{g}_{u} \tilde{g}_{d} M_{2}\right) \mu\right] .
\end{aligned}
$$

Here $M_{1,2}$ and $\mu$ are the gaugino and higgsino masses, $h_{t}$ is the top-Yukawa coupling, and $\tilde{g}_{u, d}, \tilde{g}_{u, d}^{\prime}$ are the gaugino-higgsino Yukawa couplings defined as in ref. [2]. Notice that the term proportional to $M_{1,2}$ and $\mu$ always gives a negative contribution to eq. (2). Therefore, if $m^{2}$ starts positive at the scale $\tilde{m}$, it will remain positive as the renormalization scale $\bar{\mu}$ is lowered. Thus below $\tilde{m}$, in the energy range of the Split-Supersymmetry effective theory, it is not possible to obtain radiative electroweak breaking. Had gaugino and higgsino masses induced the radiative breaking, we could have hoped to relate the dark-matter mass scale $\left(M_{1,2}, \mu\right)$ to the electroweak scale $\left(-m^{2} / \lambda\right)$, providing a connection that is still missing in the context of Split Supersymmetry.

The negative result on radiative breaking from gaugino and higgsino masses can be generalized. The RG evolution of the Higgs mass parameter $m^{2}$, for a generic field content, can be obtained by requiring that the one-loop effective potential is independent of the renormalization scale,

$$
\begin{gathered}
16 \pi^{2} \bar{\mu} \frac{d}{d \bar{\mu}} m^{2}=2 \gamma_{h} m^{2}+\Delta \\
\Delta=\left.\frac{1}{2} \frac{d^{2}}{d h^{2}} \operatorname{STr}\left(\mathcal{M}^{\dagger} \mathcal{M}\right)^{2}\right|_{h=0} .
\end{gathered}
$$

Here $\gamma_{h}=-16 \pi^{2}(\bar{\mu} / h)(d h / d \bar{\mu})$ is the Higgs anomalous dimension and $\mathcal{M}$ is the mass matrix of all particles in the Higgs background. The term proportional to $\gamma_{h}$ in eq. (33) gives a multiplicative renormalization of $m^{2}$ and therefore cannot reverse the sign of $m^{2}$ during the $\mathrm{RG}$ evolution, and cannot induce radiative breaking. The second term in eq. (3) can give an 
additive renormalization and, if positive, can trigger spontaneous symmetry breaking. For chiral fermions linearly coupled to the Higgs, we have $\mathcal{M}=A+B h$ (with generic matrices $A$ and $B)$. This gives a contribution to $\Delta$

$$
\begin{gathered}
\Delta=-2 \operatorname{Tr}\left(X^{\dagger} X+Y^{\dagger} Y\right), \\
X \equiv A B^{\dagger}+B A^{\dagger}, \quad Y \equiv \sqrt{2} A^{\dagger} B .
\end{gathered}
$$

It is apparent that eq. (5) always gives a negative contribution, and therefore fermions linearly-coupled to the Higgs cannot trigger radiative breaking.

Fermions are actually responsible for radiative breaking in Little-Higgs theories 4, but this is not in contradiction with our result. In Little-Higgs models, there are nonrenormalizable couplings between the new fermions and Higgs bilinears, of the form $\mathcal{M}=$ $A+B h^{2}$, which can lead to positive values of $\Delta$, for appropriate values of $A$ and $B$, see eq. (4). In Split Supersymmetry, we can obtain couplings of the form $\mathcal{M}=A+B h^{2}$, considering the case $M_{1,2} \gg \mu$ (or viceversa). Integrating out the gauginos, we find an effective higgsino coupling to Higgs bilinears which, through eq. (3), gives $m^{2} \propto(\alpha / \pi)\left(\mu^{3} / M\right) \ln (M / \mu)$. For an appropriate choice of the sign of the higgsino mass $\mu$, we obtain a negative contribution to the Higgs mass parameter $m^{2}$. However, in realistic models, one cannot explain why other, parametrically larger, effects (such as $m^{2} \propto(\alpha / \pi) M^{2} \ln (\tilde{m} / M)$, not to mention the boundary condition of $m^{2}$ at the scale $\tilde{m}$ ) are smaller in size than the negative contribution from higgsinos.

On the other hand, above the scale $\tilde{m}$, the radiative breaking is easily achieved [5] by the positive contribution to $\Delta$ from the stop

$$
\Delta_{\tilde{t}}=6 h_{t}^{2}\left(\tilde{m}_{Q_{3}}^{2}+\tilde{m}_{U_{3}}^{2}\right)
$$

where $\tilde{m}_{Q_{3}, U_{3}}$ are the $\tilde{t}_{L, R}$ soft masses, of the order of $\tilde{m}$. This leads to $m^{2}=\mathcal{O}\left(-\tilde{m}^{2}\right)$, which has to be tamed by the tuning condition. From our discussion, it is now manifest the meaning of the tuning: it corresponds to the condition that the Higgs mass parameter $m^{2}$ changes sign precisely at the scale $\tilde{m}$, at which the squarks are integrated out. As shown before, the running below $\tilde{m}$ has a modest impact on $m^{2}$ and, in particular, it does not further change its sign.

The supersymmetric Higgs potential above $\tilde{m}$, along the real and neutral components, is

$$
V=\frac{m_{H_{u}}^{2}}{2} H_{u}^{2}+\frac{m_{H_{d}}^{2}}{2} H_{d}^{2}-B_{\mu} H_{u} H_{d}+\frac{g^{2}+g^{\prime 2}}{32}\left(H_{u}^{2}-H_{d}^{2}\right)^{2},
$$




\begin{tabular}{|c|c|c|}
\hline$\tilde{m}(\mathrm{GeV})$ & $K \sin ^{2} \beta$ & $\omega$ \\
\hline \hline $10^{4}$ & 0.28 & 0.024 \\
$10^{7}$ & 0.19 & 0.020 \\
$10^{10}$ & 0.12 & 0.016 \\
$10^{13}$ & 0.06 & 0.009 \\
\hline
\end{tabular}

Table 1: Values of $K \sin ^{2} \beta$ (this combination is almost independent of $\tan \beta$ ) and $\omega$ for different values of $\tilde{m}$ and for $m_{t}^{\text {pole }}=178 \mathrm{GeV}$.

with mass parameters of the order of $\tilde{m}^{2}$. It is convenient to rewrite the potential in terms of the fields

$$
\left(\begin{array}{l}
h \\
H
\end{array}\right)=\left(\begin{array}{cc}
\cos \beta & \sin \beta \\
-\sin \beta & \cos \beta
\end{array}\right)\left(\begin{array}{l}
H_{d} \\
H_{u}
\end{array}\right), \quad \sin 2 \beta \equiv \frac{2 B_{\mu}}{m_{H_{u}}^{2}+m_{H_{d}}^{2}}
$$

We obtain

$$
\begin{gathered}
V=\frac{\lambda}{8}\left(h^{2}+2 \frac{m^{2}}{\lambda}-2 \tan 2 \beta h H-H^{2}\right)^{2}+\frac{m_{H_{u}}^{2}+m_{H_{d}}^{2}}{2} H^{2}, \\
\lambda=\frac{g^{2}+g^{\prime 2}}{4} \cos ^{2} 2 \beta, \quad m^{2}=\frac{\left(t^{2}-1\right)}{\left(t^{2}+1\right)^{2}}\left(m_{H_{u}}^{2} t^{2}-m_{H_{d}}^{2}\right), \quad t \equiv \tan \beta .
\end{gathered}
$$

For $m_{H_{u}}^{2}+m_{H_{d}}^{2}=\mathcal{O}\left(\tilde{m}^{2}\right)$, the field $H$ decouples at the squark-mass scale. The tuning condition corresponds to choosing $\mathrm{m}^{2}$ approximately zero (and slightly negative). Once this condition is applied, the potential of the effective theory coincides with eq. (11), with the boundary conditions at $\tilde{m}$ given by eq. (11).

We want to investigate what information the tuning condition can provide us on the fundamental parameters at a large scale $M_{X}$, which we will identify with the GUT scale. The relations between the soft masses at the scale $M_{X}$ (denoted by a bar) and those at the matching scale $\tilde{m}$ are

$$
\begin{aligned}
m_{H_{u}}^{2}(\tilde{m}) & =\bar{m}_{H_{u}}^{2}-K\left(\bar{m}_{H_{u}}^{2}+\bar{m}_{Q_{3}}^{2}+\bar{m}_{U_{3}}^{2}\right)-\omega \bar{S} \\
m_{H_{d}}^{2}(\tilde{m}) & =\bar{m}_{H_{d}}^{2}+\omega \bar{S} \\
m_{Q_{3}}^{2}(\tilde{m}) & =\bar{m}_{Q_{3}}^{2}-\frac{K}{3}\left(\bar{m}_{H_{u}}^{2}+\bar{m}_{Q_{3}}^{2}+\bar{m}_{U_{3}}^{2}\right)-\frac{\omega}{3} \bar{S} \\
m_{U_{3}}^{2}(\tilde{m}) & =\bar{m}_{U_{3}}^{2}-\frac{2 K}{3}\left(\bar{m}_{H_{u}}^{2}+\bar{m}_{Q_{3}}^{2}+\bar{m}_{U_{3}}^{2}\right)+\frac{4 \omega}{3} \bar{S} \\
\bar{S} & \equiv \bar{m}_{H_{u}}^{2}-\bar{m}_{H_{d}}^{2}+\sum_{i=1}^{3}\left(\bar{m}_{Q_{i}}^{2}-2 \bar{m}_{U_{i}}^{2}+\bar{m}_{D_{i}}^{2}-\bar{m}_{L_{i}}^{2}+\bar{m}_{E_{i}}^{2}\right) .
\end{aligned}
$$

Here we have neglected terms of weak-scale size $(M, \mu, A)$ and kept only the one-loop leading contributions from gauge and top-Yukawa couplings (which is adequate for not too 

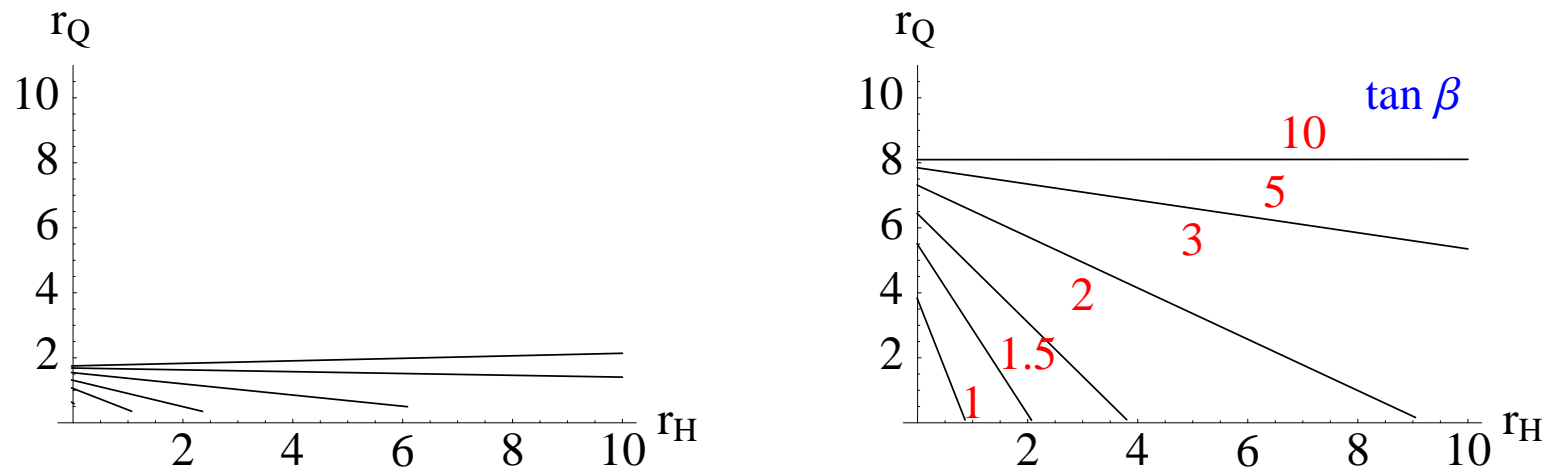

Figure 1: The relation between $r_{Q}$ and $r_{H}$ necessary to allow for tuning of the weak scale, in the case of SU(5) GUT boundary conditions. We have taken $\tilde{m}=10^{6} \mathrm{GeV}\left(10^{13} \mathrm{GeV}\right)$ in the left (right) panels, and $\tan \beta$ varying from 1 (lower line) to 10 (upper line).

large values of $\tan \beta$ ). The coefficients $K$ and $\omega$ are given by

$$
\begin{gathered}
K=\left.\frac{3 F \lambda_{t}^{2}}{16 \pi^{2} E}\right|_{\tilde{m}} E(\bar{\mu})=\prod_{i=1}^{3}\left[\frac{g_{i}^{2}\left(M_{X}\right)}{g_{i}^{2}(\bar{\mu})}\right]^{\frac{2 c_{i}}{b_{i}}} \quad F=\int_{0}^{\ln M_{X}^{2} / \tilde{m}^{2}} d\left(\ln M_{X}^{2} / \bar{\mu}^{2}\right) E \\
\omega=\frac{3 g_{1}^{2}(\tilde{m})}{160 \pi^{2}} \ln \frac{M_{X}^{2}}{\tilde{m}^{2}},
\end{gathered}
$$

where $c_{i}=(13 / 30,3 / 2,8 / 3), b_{i}=(33 / 5,1,-3)$, and $g_{1}$ has GUT normalization. The numerical values of $K$ and $\omega$ for different $\tilde{m}$ are given in table 1. In eq. (17) $\lambda_{t}$ is the supersymmetric top-Yukawa coupling related to the low-energy coupling $h_{t}$ by the matching condition $\lambda_{t}(\tilde{m}) \sin \beta=h_{t}(\tilde{m})$. The parameter $K$ has a maximum value $(K<1 / 2)$, once we require the absence of Landau poles below the scale $M_{X}$.

The conditions for tuning the electroweak scale are

$$
\begin{aligned}
& m^{2}(\tilde{m}) \equiv \frac{\left(t^{2}-1\right)}{\left(t^{2}+1\right)^{2}}\left[m_{H_{u}}^{2}(\tilde{m}) t^{2}-m_{H_{d}}^{2}(\tilde{m})\right]=0 \\
& m_{H_{u}}^{2}(\tilde{m})+m_{H_{d}}^{2}(\tilde{m})>0 \\
& m_{Q_{3}}^{2}(\tilde{m})>0 \\
& m_{U_{3}}^{2}(\tilde{m})>0 .
\end{aligned}
$$

Equations (201)-(22) correspond to the requirement that no electric-charge or colour breaking minima are developed at the scale $\tilde{m}$, as recently discussed in ref. 6].

Let us consider the case in which the soft masses satisfy SU(5) GUT boundary conditions, $\bar{m}_{Q}=\bar{m}_{U}=\bar{m}_{E}$ and $\bar{m}_{D}=\bar{m}_{L}$. This case can be realized when supersymmetry is broken in a hidden sector and communicated at a scale larger than $M_{X}$. Then, in terms of $r_{Q}=\bar{m}_{Q_{3}}^{2} / \bar{m}_{H_{u}}^{2}$ 
and $r_{H}=\bar{m}_{H_{d}}^{2} / \bar{m}_{H_{u}}^{2}$, eqs. (19)-(22) are equivalent to ${ }^{1}$

$$
\begin{gathered}
2 K t^{2} r_{Q}=\left[\omega\left(t^{2}+1\right)-1\right] r_{H}+t^{2}(1-K-\omega)-\omega \\
-\frac{\omega}{1-\omega}<r_{H}<\frac{t^{2}[3(1-\omega)-K(7-12 \omega)]-(3-4 K) \omega}{(3-4 K)(1-\omega)-3 t^{2} \omega(1-4 K)} .
\end{gathered}
$$

The allowed region of $r_{Q}-r_{H}$ parameters is shown in fig. 1, for characteristic values of $\tilde{m}$ and $\tan \beta$. The tuning of the electroweak scale can be achieved in a large area of parameters for $r_{Q}$ and $r_{H}$ of order unity. Natural values of the boundary conditions are compatible with the breaking.

As apparent from fig. 11, the available area of $r_{Q}-r_{H}$ shrinks as $\tan \beta$ is lowered. Indeed, from eq. (24), we obtain that a solution for $r_{H}$ exists only if

$$
K<\frac{3}{7}\left(\frac{1-2 \omega}{1-\frac{19}{7} \omega}\right) .
$$

This bound is more stringent than the constraint $K<1 / 2$ (from absence of Landau poles). Therefore Split Supersymmetry with SU(5) GUT boundary conditions cannot have the top Yukawa coupling at the infrared fixed point ${ }^{2}$. The origin of the bound in eq. (25) can be easily traced. Larger values of $K$ require smaller values of $r_{Q}$ to tune the weak scale, see eq. (19). But if $r_{Q}$ is too small, eq. (22) can no longer be satisfied and the stop mass square becomes negative.

An upper bound on $K$ corresponds to an upper bound on $\lambda_{t}(\tilde{m})$, see eq. (17)). Since $\lambda_{t}(\tilde{m})=h_{t}(\tilde{m}) / \sin \beta$, eq. (25) gives a lower bound on $\tan \beta$. This bound in shown in fig. 2. We fix $\alpha_{s}\left(M_{Z}\right)=0.119$ and, because of the significant dependence on the top-Yukawa coupling, we show the result for three values of the pole mass, $m_{t}=178 \pm 4 \mathrm{GeV}$. Since we are considering general boundary conditions for the Higgs soft masses (i.e. $r_{H} \neq 1$ ), $\tan \beta$ can in principle be smaller than 1 . Low values of $\tan \beta$ help in obtaining bottom-tau unification. The lower limit on $\tan \beta$ can be translated into a lower limit on the Higgs mass, as shown in fig. 2 .

We also remark that SU(5) GUT boundary conditions allow for the possibility of a double fine-tuning in which both the weak scale and the charged Higgs mass $\left(m_{H_{u}}^{2}+m_{H_{d}}^{2}\right)$ are much smaller than $\tilde{m}$, and therefore the low-energy theory has two Higgs doublets. This is obtained for

$$
r_{H}=-\frac{\omega}{1-\omega}, \quad r_{Q}=\frac{1-K-\omega(2-K)}{2 K(1-\omega)},
$$

\footnotetext{
${ }^{1}$ When $t^{2}>(3-4 K)(1-\omega) /[3 \omega(1-4 K)]$ and $K<1 / 4$, the upper bound on $r_{H}$ disappears. This has no consequences for our discussion.

${ }^{2}$ The infrared fixed point of the top Yukawa coupling in Split Supersymmetry was recently studied in ref. [7].
} 

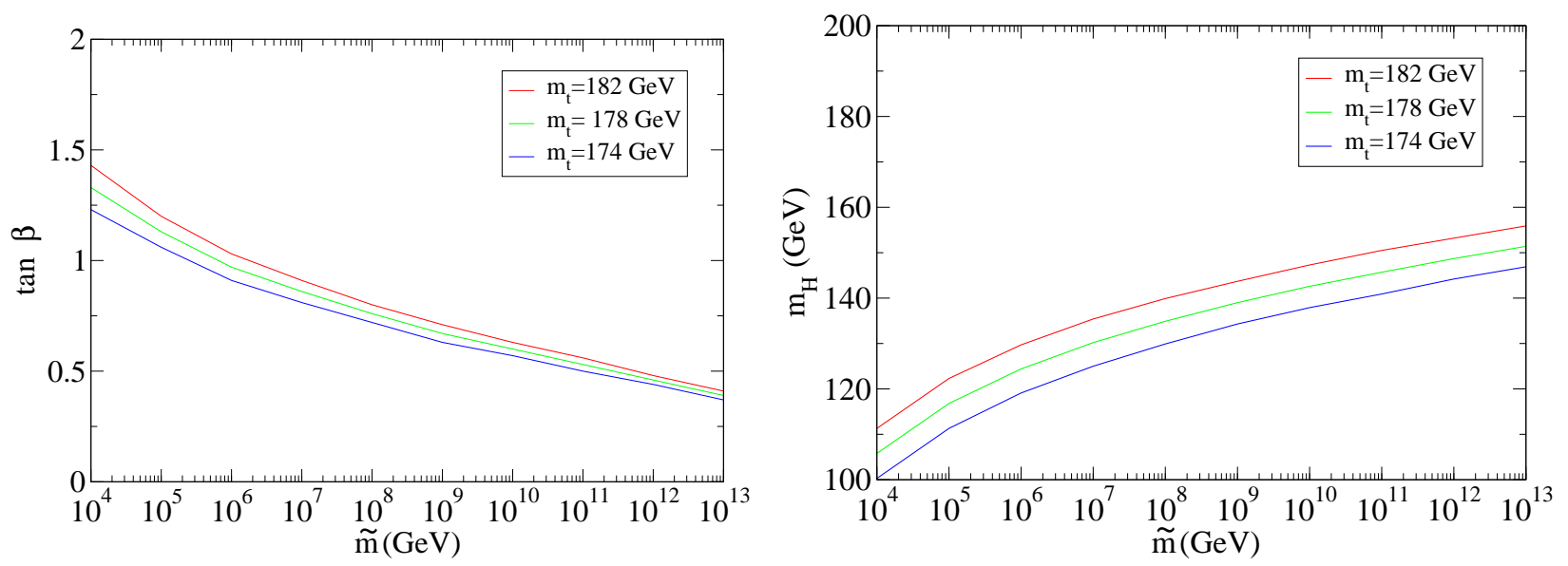

Figure 2: Lower bound on $\tan \beta$ (left) and on $m_{H}$ (right) obtained by requiring weak-scale tuning, in the case of $\mathrm{SU}(5)$ GUT boundary conditions. The different lines correspond to $m_{t}^{\text {pole }}=174,178,182 \mathrm{GeV}$.

which corresponds to the border line of the area in fig. 1 at low $r_{H}$. However, the double fine-tuning requires a peculiar boundary condition, with $r_{H}$ very small and negative.

With stronger assumptions on the soft masses, we can obtain sharper predictions. Let us consider the universality hypothesis, which implies $r_{Q}=r_{H}=1$. In Split Supersymmetry, universality is not necessary to solve the flavour problem, but it could appear in particular schemes of supersymmetry breaking. In this case, the tuning of the weak scale leads to a prediction of the value of $\tan \beta$

$$
\tan \beta=\frac{1}{\sqrt{1-3 K}}
$$

which is shown in fig. 3. In Split Supersymmetry with universal boundary conditions, the Higgs mass is uniquely predicted, as a function of $\tilde{m}$, as shown in fig. 3. The band in fig. 3. corresponds to the uncertainty in $m_{t}$ and therefore the prediction will be further sharpened, if the experimental error in the top mass is reduced.

Finally, it is important to mention that, if we abandon SU(5) GUT boundary conditions and consider the most general pattern of soft terms, the lower bound in eq. (25) can be evaded and the top-Yukawa infrared fixed point can be reached. Indeed, for arbitrary soft terms, even when $\bar{S}=0$, we can tune the weak scale for any value of $\tan \beta$ and any value of $K$ (with $0<K<1 / 2)$ as long as $r_{H}<t^{2}(1-2 K) /(1-K)$.

The reason why the tuning of the weak scale can be obtained so easily for natural initial values of the soft masses and even for universal boundary conditions lies on the efficiency of the radiative breaking in supersymmetry and, ultimately, in the favourable observed value of the top mass. The possibility of reproducing the weak scale for large scalar soft masses with 

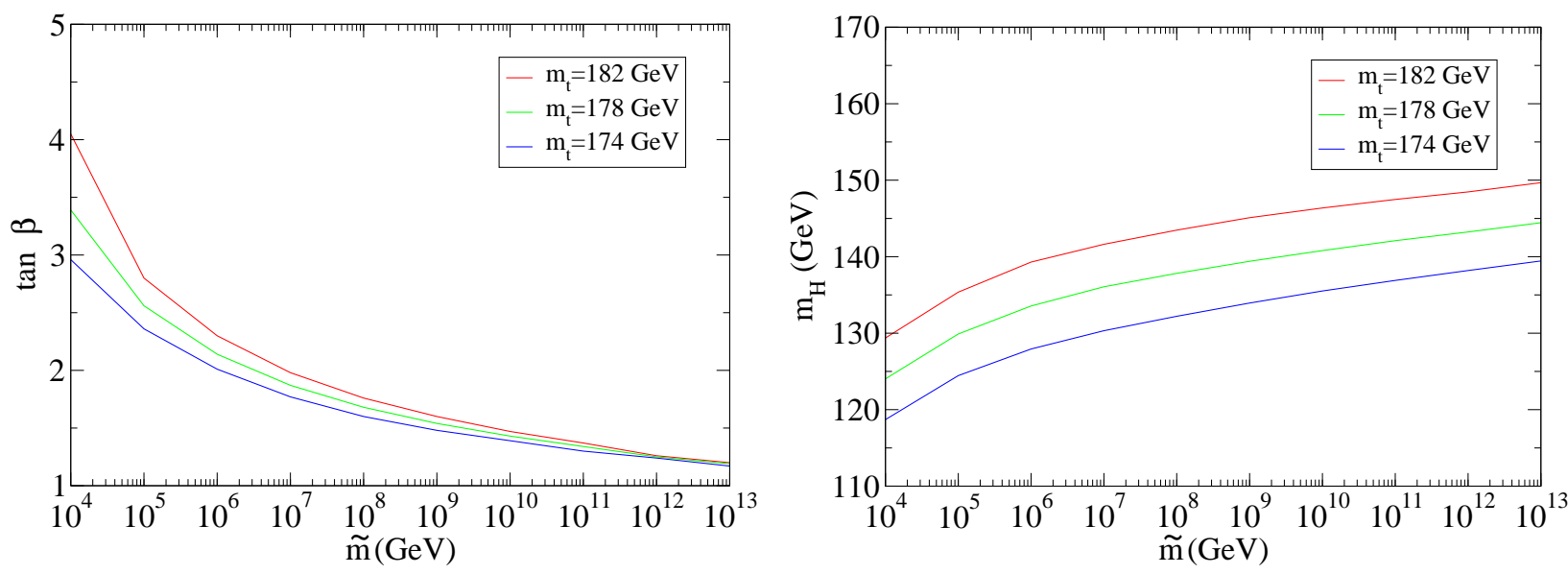

Figure 3: Values of $\tan \beta$ (left) and $m_{H}$ (right) obtained by requiring weak-scale tuning, in the case of universal boundary conditions. The different lines correspond to $m_{t}^{\text {pole }}=174$, 178, $182 \mathrm{GeV}$.

universal boundary conditions (first observed in ref. [8]) was carefully studied in ref. [9], where it was named "focus point". Split Supersymmetry brings that possibility to the extreme consequences.

In conclusion, we have discussed the meaning of the tuning condition in Split Supersymmetry and shown how it leads to interesting information both on the underlying soft masses and on low-energy measurable observables, like the Higgs mass $m_{H}$ and $\tan \beta$. Given specific high-energy matching conditions, one can make testable predictions. In particular, we have shown that $\mathrm{SU}(5)$ GUT boundary conditions imply a lower bound on $\tan \beta$ and on $m_{H}$, while universal boundary conditions imply a determination of $\tan \beta$ and $m_{H}$, as functions of $\tilde{m}$. Viceversa, measurements of the low-energy parameters can help us discriminating among underlying models of supersymmetry breaking.

We thank N. Arkani-Hamed, R. Rattazzi and A. Romanino for very useful discussions.

\section{References}

[1] N. Arkani-Hamed and S. Dimopoulos, arXiv:hep-th/0405159.

[2] G. F. Giudice and A. Romanino, Nucl. Phys. B 699 (2004) 65 [Erratum-ibid. B 706 (2005) 65] arXiv:hep-ph/0406088.

[3] N. Arkani-Hamed, S. Dimopoulos, G. F. Giudice and A. Romanino, Nucl. Phys. B 709 (2005) 3 arXiv:hep-ph/0409232. 
[4] N. Arkani-Hamed, A. G. Cohen and H. Georgi, Phys. Lett. B 513 (2001) 232 arXiv:hep-ph/0105239.

[5] L. E. Ibanez and G. G. Ross, Phys. Lett. B 110 (1982) 215.

[6] A. Ibarra, arXiv:hep-ph/0503160.

[7] K. Huitu, J. Laamanen, P. Roy and S. Roy, arXiv:hep-ph/0502052.

[8] R. Barbieri and G. F. Giudice, Nucl. Phys. B 306 (1988) 63.

[9] J. L. Feng, K. T. Matchev and T. Moroi, Phys. Rev. D 61 (2000) 075005 arXiv:hep-ph/9909334; J. L. Feng, K. T. Matchev and F. Wilczek, Phys. Lett. B 482 (2000) 388 arXiv:hep-ph/0004043|; J. L. Feng and K. T. Matchev, Phys. Rev. D 63 (2001) 095003 arXiv:hep-ph/0011356. 